\title{
14-3-3 $\sigma$ is an independent prognostic biomarker for gastric cancer and is associated with apoptosis and proliferation in gastric cancer
}

\author{
YI-LIANG LI ${ }^{1 *}$, LIHUA LIU $^{2 *}$, YANG XIAO ${ }^{3}$, TAO ZENG $^{4}$ and $\mathrm{CHAO} \mathrm{ZENG}^{5}$ \\ ${ }^{1}$ Department of Neurology, The Central Hospital of Loudi Affiliated to the University of South China, Loudi, Hunan 417000; \\ ${ }^{2}$ Department of Respiratory Disease, The First Affiliated Hospital of Guangxi Medical University, Nanning, \\ Guangxi 530021; ${ }^{3}$ Department of Orthopaedics, The Central Hospital of Loudi Affiliated to the University of South China, \\ Loudi, Hunan 417000; ${ }^{4}$ School of Laboratory Medicine and ${ }^{5}$ Department of Pathology, \\ Guangdong Medical College, Dongguan, Guangdong 523808, P.R. China
}

Received February 3, 2014; Accepted September 26, 2014

DOI: $10.3892 / \mathrm{ol} .2014 .2676$

\begin{abstract}
. 14-3-3 proteins participate in various cellular processes, including apoptosis, proliferation and malignant transformation. 14-3-3 $\sigma$, a member of the 14-3-3 protein family, is important in several types of cancer; however, little is known about the clinical significance and biological roles of 14-3-3 $\sigma$ in gastric cancer. The present study analyzed the expression pattern of 14-3-3 $\sigma$ in gastric cancer and investigated its correlation with the prognosis of gastric cancer patients. Furthermore, the association of 14-3-3 $\sigma$ with $\mathrm{Ki}-67, \mathrm{Bcl}-2$ and Bax was evaluated. 14-3-3 $\sigma$ was expressed at higher level in gastric cancer tissue compared with healthy gastric tissue, and 14-3-3 $\sigma$ expression was significantly correlated with tumor size and tumor node metastasis stage $(\mathrm{P}<0.05)$. To the best of our knowledge, the present study data are the first to suggest that 14-3-3 $\sigma$ expression has been significantly associated with poor prognosis in gastric cancer. Additionally, 14-3-3 $\sigma$ overexpression was positively correlated with Ki-67 and Bcl-2 expression levels. Thus, $14-3-3 \sigma$ is a potential prognostic marker for gastric cancer patients, and may be involved in regulating the apoptosis and proliferation of gastric cancer cells.
\end{abstract}

\section{Introduction}

Despite the declining incidence of gastric cancer in certain parts of the world, it remains one of the leading causes of cancer-related mortality worldwide (1). Gastric cancer is hypothesized to develop in a multi-step process that includes

Correspondence to: Dr Chao Zeng, Department of Pathology, Guangdong Medical College, 1 Xincheng Road, Dongguan, Guangdong 523808, P.R. China

E-mail: zengchaosysu@126.com

${ }^{*}$ Contributed equally

Key words: 14-3-36, prognosis, apoptosis, proliferation, immunohistochemistry the activation and overexpression of oncogenes, such as K-sam and c-Met (2,3), as well as the inactivation of tumor suppressor genes, such as APC (4). Various genes are associated with stomach cancer, such as TP53 (5), TGF- $\beta$ (6) and Runx3 (7); however, their association with gastric cancer is weak. Therefore, there is a requirement to identify novel molecular markers to improve the prognosis of gastric cancer patients.

The 14-3-3 protein family has seven distinct 14-3-3 genes, denoted $\beta, \varepsilon, \gamma, \zeta, \sigma, \eta$ and $\tau$. Thus far, 14-3-3 proteins have been identified to participate in the biological regulation of cell behavior such as apoptosis, the cell cycle and malignant transformation, by interacting with ligands $(8,9)$. Of the seven 14-3-3 genes, 14-3-3 $\sigma$ has been identified as an epithelial-specific marker and is associated with $\mathrm{G} 2 / \mathrm{M}$ checkpoint control in the cell cycle (10); 14-3-3 $\sigma$ induced the activation of tumor protein p53, and bound to cyclin-dependent kinase-2 (CDK2) and -4 (CDK4) (11). Thus, 14-3-3 $\sigma$ may be defined as a negative regulator of the cell cycle. Protein expression levels of 14-3-3 $\sigma$ are significantly reduced or negligible in various types of primary cancer of epithelial origin, including lung (12), prostate (13) and bladder carcinoma (14). This cancer-related inactivation of

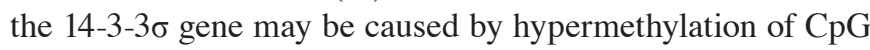

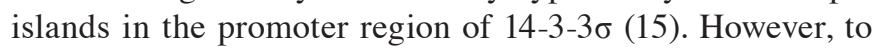
date, few studies have reported a correlation between 14-3-3o expression levels and clinicopathological features of gastric cancer. Therefore, to investigate the clinical significance and prognostic value of 14-3-3 $\sigma$ in gastric cancer, the present study analyzed 14-3-3 $\sigma$ expression in 60 gastric cancer samples and evaluated its correlation with specific clinical outcomes of gastric cancer patients.

\section{Materials and methods}

Patients and tissue samples. Sixty paraffin-embedded gastric cancer samples were collected from The Central Hospital of Loudi Affiliated to the University of South China (Loudi, China), between 2007 and 2008. None of the patients had received preoperative anticancer treatment. Clinicopathological features, including age, gender, tumor differentiation degree, tumor volume, tumor invasion depth, tumor node metastasis (TNM) stage and lymph node metastasis are detailed in Table I. 
Informed consent was obtained from each patient upon collection of the samples. The present study was approved by the institutional research medical ethics committee of The Central Hospital of Loudi Affiliated to the University of South China.

Tissue microarray and immunohistochemistry. Tissue microarrays (TMAs) were constructed as previously described (16). Briefly, paraffin-embedded donor tissue blocks and the corresponding hematoxylin and eosin-stained slides were overlaid for TMA sampling. Cylindrical tissue samples (diameter, $0.6 \mathrm{~mm}$ ) were punctured in triplicate from specific areas of the donor tissue and re-embedded into a recipient paraffin block at the designated location.

Routine techniques were utilized to cut $4-\mu \mathrm{m}$ sections from the deparaffinized TMAs. The slides were microwaved in citrate buffer for $8 \mathrm{~min}$ for antigen retrieval, and

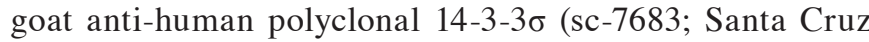
Biotechnology, Inc., Santa Cruz, CA, USA) was applied as the primary antibody in a 1:150 dilution. Goat anti-human monoclonal Ki-67 (GT209401), goat anti-human polyclonal Bcl-2 (GM088701) and goat anti-human Bax (A353302) were purchased from Gene Biotechnology (Shanghai, China). Labeling was detected by horseradish peroxidase-conjugated mouse anti-goat IgG and staining with 3,3'-diaminobenzidine (all Maxim-Bio, Inc., Fuzhou, China). Finally, the slides were counterstained with hematoxylin. 14-3-3 $\sigma, \mathrm{Bcl}-2$ and Bax were scored according to the staining intensity ( 0 , no staining; 1, weak staining; 2 , moderate staining; 3 , strong staining) and the percentage of positively stained tumor cells $(0,0 \%$ of tumor cells stained; $1,1-9 \%$ of tumor cells stained; $2,10-50 \%$ of tumor cells stained; 3, 51-75\% of tumor cells stained; $4,>75 \%$ of tumor cells stained). If the product of the staining intensity and the percentage of positively stained tumor cells was $\geq 2$, the staining was considered to be positive (+). Ki-67 was scored according to the number of positive gastric cancer cells. A negative control was performed by replacing the primary antibody with the appropriate serum controls.

Statistical analysis. All statistical analyses were performed using SPSS for Windows (version 13.0; SPSS, Inc., Chicago, IL, USA). The correlation between 14-3-3 $\sigma$ expression rates and clinicopathological features of gastric cancer cases was evaluated using Fisher's exact test. Multivariate analysis was performed using Cox proportional-hazards regression. Overall survival curves were generated according to the Kaplan-Meier method. $\mathrm{P}<0.05$ was considered to indicate a statistically significant difference.

\section{Results}

Expression of 14-3-3o in gastric cancer. To investigate the expression of 14-3-3 $\sigma$ in gastric cancer, immunohistochemical staining for 14-3-3 $\sigma$ was performed on a TMA containing 60 pairs of gastric cancer samples and the corresponding healthy gastric mucosa tissues. In the present study, compared with weak or negative expression in normal gastric mucosal tissues, $63.3 \%(38 / 60)$ of gastric cancer cases exhibited posi-

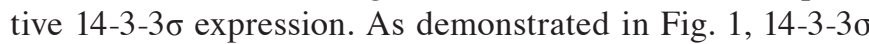
staining was evident in the cytoplasm of poorly, moderately and well-differentiated gastric cancer cells.
Table I. Correlation between 14-3-3 $\sigma$ expression rate and various clinicopathological features in 60 cases of gastric cancer.

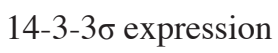

Variable Cases, Negative Positive P-value

$\mathrm{n}$

\begin{tabular}{lrrrr}
\hline $\begin{array}{l}\text { Gender } \\
\text { Male }\end{array}$ & 31 & 15 & 16 & 0.064 \\
Female & 29 & 7 & 22 & \\
Age, years & & & & \\
$\geq 60$ & 28 & 11 & 17 & 0.791 \\
$<60$ & 32 & 11 & 21 &
\end{tabular}

Tumor size, $\mathrm{cm}$

$\begin{array}{lrrrr}\geq 5 & 26 & 5 & 21 & 0.017^{\mathrm{a}} \\ <5 & 34 & 17 & 17 & \\ \text { Differentiation degree } & & & & \\ \quad \text { Well/Moderately } & 39 & 13 & 26 & 0.577 \\ \quad \text { Poorly } & 21 & 9 & 12 & \\ \text { Invasion depth } & & & & \\ \text { T1 + T2 } & 41 & 18 & 23 & 0.149 \\ \text { T3 + T4 } & 19 & 4 & 15 & \\ \text { TNM stage } & & & & \\ \text { I + II } & 34 & 18 & 16 & 0.003^{\mathrm{a}} \\ \text { III + IV } & 26 & 4 & 22 & \end{array}$

Lymph node

metastasis

$\begin{array}{lrrrr}\text { Yes } & 37 & 17 & 20 & 0.097 \\ \text { No } & 23 & 5 & 18 & \end{array}$

${ }^{\mathrm{a}} \mathrm{P}<0.05$. TNM, tumor node metastasis.

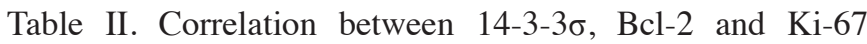
expression.

\begin{tabular}{lcccc}
\hline & & \multicolumn{2}{c}{ Bcl-2 staining } & \\
\cline { 3 - 4 } $\begin{array}{l}\text { 14-3-3 } \sigma \\
\text { staining }\end{array}$ & Cases, $\mathrm{n}$ & Positive & Negative & $\begin{array}{c}\text { Ki-67, } \\
\%( \pm \mathrm{SD})\end{array}$ \\
\hline Positive & 38 & $25^{\mathrm{a}}$ & 13 & $48.1 \pm 5.1^{\mathrm{a}}$ \\
Negative & 22 & 8 & 14 & $17.6 \pm 4.8$ \\
\hline
\end{tabular}

${ }^{\mathrm{a}} \mathrm{P}<0.05$, vs. negative $14-3-3 \sigma$ staining. SD, standard deviation.

Association between 14-3-3 $\sigma$ expression and clinicopathological features of gastric cancer. Table I indicates the expression rates of 14-3-3 $\sigma$ in gastric cancer with respect to various clinicopathological features. The expression rates of 14-3-3 $\sigma$ were significantly higher in patients exhibiting larger tumors (size, $\geq 5 \mathrm{~cm} ; \mathrm{P}=0.017)$ and an advanced TNM stage $(\mathrm{P}=0.003)$. 
A

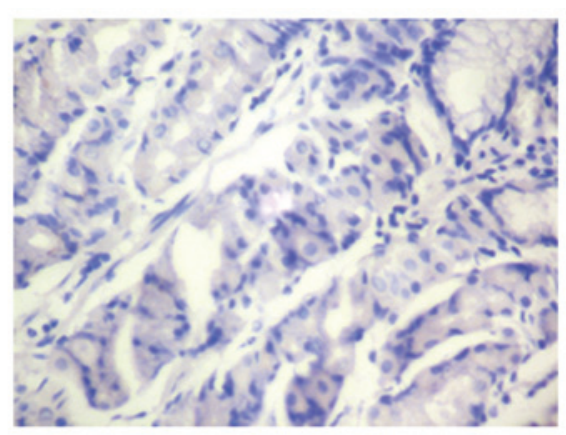

C

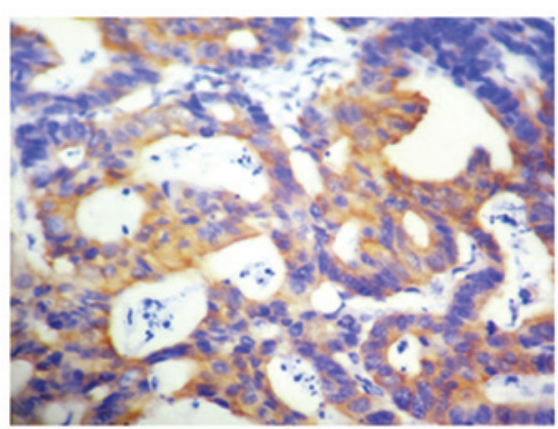

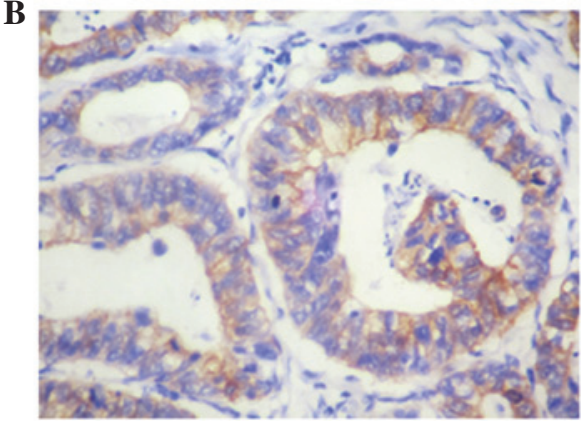

D

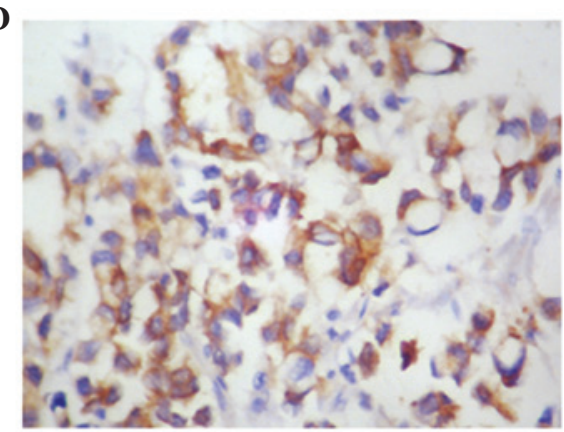

Figure 1. Expression pattern of 14-3-3 $\sigma$ in gastric cancer. (A) No 14-3-3 $\sigma$ expression in healthy gastric tissues. (B) Overexpression of 14-3-3 $\sigma$ in the cytoplasm of well-differentiated gastric cancer cells. (C) High 14-3-3 $\sigma$ expression in moderately differentiated gastric cancer cells. (D) Positive 14-3-3 $\sigma$ expression in poorly differentiated gastric cancer cells (magnification, $\mathrm{x} 200$ ).
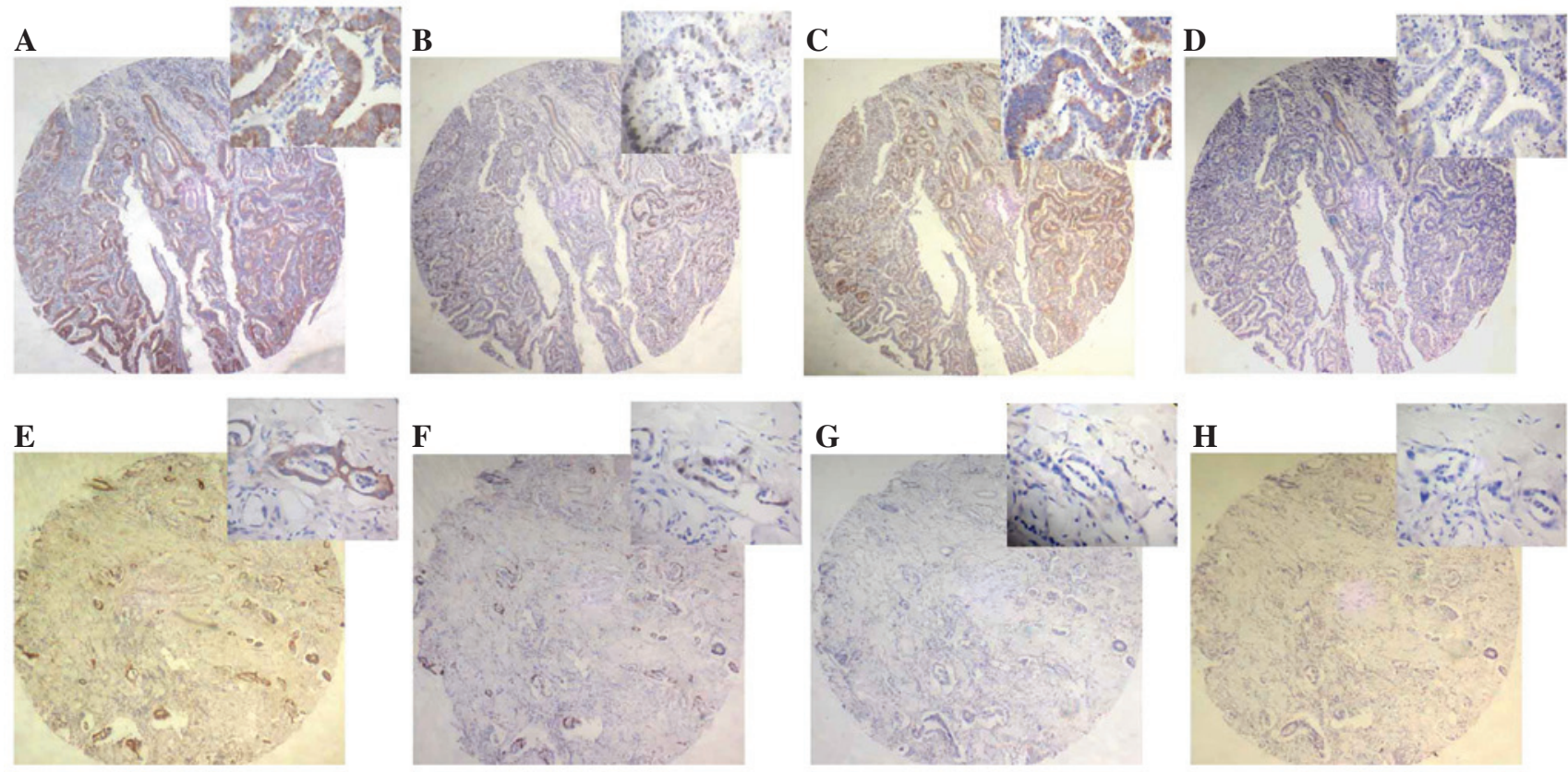

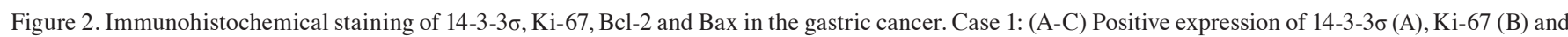

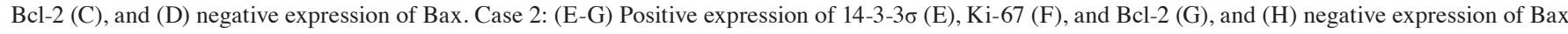
(low power magnification, x100; high power magnification, x200).

However, no significant difference was identified between 14-3-30 expression and the other clinicopathological features investigated, such as age, gender, tumor differentiation degree, depth of tumor invasion and lymph node metastasis $(\mathrm{P}>0.05)$.

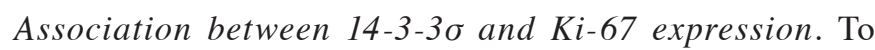
explore the influence of $14-3-3 \sigma$ on the proliferation of gastric cancer cells, the present study analyzed the correlation between 14-3-3 $\sigma$ and $\mathrm{Ki}-67$ expression. The percentage of cells exhibiting positive Ki-67 expression was significantly higher $(48.1 \pm 5.1)$ in the $14-3-3 \sigma$ positive expression group compared with the 14-3-3 $\sigma$ negative expression group $(17.6 \pm 4.8)$ (Table II). These findings indicate that 14-3-3 $\sigma$ may participate in tumor cell proliferation. 
Table III. Univariate and multivariate analyses of overall survival in gastric cancer patients.

\begin{tabular}{|c|c|c|c|c|c|c|}
\hline \multirow[b]{2}{*}{ Variable } & \multicolumn{3}{|c|}{ Univariate analysis } & \multicolumn{3}{|c|}{ Multivariate analysis } \\
\hline & Hazard ratio & $95 \% \mathrm{CI}$ & P-value & Hazard ratio & $95 \% \mathrm{CI}$ & P-value \\
\hline Gender & 1.561 & $0.832-3.914$ & 0.372 & & & \\
\hline Age, year & 1.126 & $0.982-1.145$ & 0.541 & & & \\
\hline Invasion depth & 5.530 & $2.479-13.885$ & $0.001^{\mathrm{a}}$ & 2.726 & $0.789-6.543$ & 0.054 \\
\hline Differentiation degree & 0.986 & $0.323-2.879$ & 0.788 & & & \\
\hline Tumor size & 1.013 & $0.346-3.190$ & 0.977 & & & \\
\hline TNM stage & 0.104 & $0.032-0.501$ & $0.003^{\mathrm{a}}$ & 0.195 & $0.040-0.765$ & $0.026^{\mathrm{a}}$ \\
\hline Lymph node metastasis & 6.114 & $3.062-18.767$ & $0.002^{\mathrm{a}}$ & 3.378 & $1.483-9.323$ & $0.013^{\mathrm{a}}$ \\
\hline $14-3-3 \sigma$ expression & 5.983 & $2.690-17.654$ & $0.002^{\mathrm{a}}$ & 3.896 & $1.719-9.891$ & $0.028^{\mathrm{a}}$ \\
\hline
\end{tabular}

${ }^{\mathrm{a}} \mathrm{P}<0.05$. CI, confidence interval; TNM, tumor node metastasis.

Association between 14-3-3 $\sigma$ and Bcl-2/Bax expression. To identify the effect of 14-3-3 $\sigma$ on the apoptosis of gastric cancer cells, the present study investigated the correlation between 14-3-3 $\sigma$ and Bcl-2/Bax expression. Bcl-2 staining was identified in 25/38 cases of positive 14-3-3 $\sigma$ expression (Table II). As demonstrated in Fig. 2, Bcl-2 expression was predominantly present within the cytoplasm of gastric cancer cells. Furthermore, 14/22 cases of negative 14-3-3 $\sigma$ expression did not exhibit Bcl-2 staining (Table II). However, with the exception of six positive expression cases, no Bax immunoreactivity was detected in these samples.

Association between 14-3-3 $\sigma$ expression and overall survival in gastric cancer. To better elucidate the effect of 14-3-3 $\sigma$ expression on the prognosis of gastric cancer, Kaplan-Meier survival curves were used to investigate the overall survival

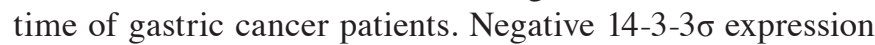
patients exhibited improved prognoses compared with positive 14-3-3 $\sigma$ expression patients (Fig. 3). Additionally, univariate and multivariate analyses were employed to assess the role of 14-3-3 $\sigma$ expression and specific clinicopathological features on the prognosis of gastric cancer patients. Univariate analysis demonstrated that lymph node metastasis, TNM stage, tumor invasion depth and 14-3-3 $\sigma$ expression were significantly associated with the overall survival of gastric cancer patients. Furthermore, multivariate analyses identified that lymph node metastasis, TNM stage and 14-3-3 $\sigma$ expression were correlated with poor overall survival of gastric cancer patients (Table III). Of the clinicopathological features investigated in the present study, lymph node metastasis was the most independent prognostic indicator of gastric cancer $(\mathrm{P}=0.013)$. Therefore, 14-3-3 $\sigma$ expression may act as a prognostic biomarker for gastric cancer.

\section{Discussion}

$14-3-3 \sigma$ is vital at the $\mathrm{G} 2 / \mathrm{M}$ checkpoint, as it sequesters the Cdc2/cyclin B1 complex; therefore, 14-3-3 $\sigma$ may be involved in the development of cancer. However, it should be considered that $14-3-3 \sigma$ exhibits different functions in the carcinogenesis

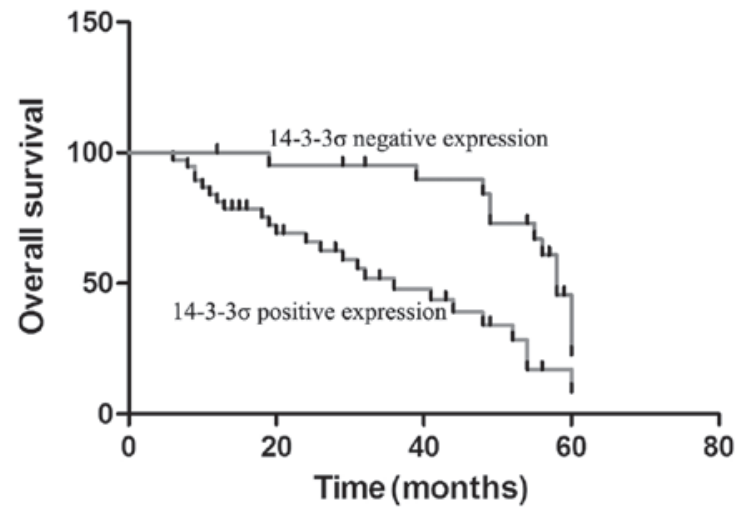

Figure 3. Kaplan-Meier survival analysis of 14-3-3 $\sigma$ expression in gastric cancer patients. Patients with negative 14-3-3 $\sigma$ expression exhibited a more

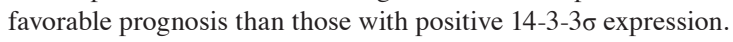

of different human organs. For example, downregulation of 14-3-3 $\sigma$ expression has been observed in colon (17), liver (18), breast (19) and ovarian cancer (20), and loss of 14-3-3 $\sigma$ expression has been associated with poor prognosis in epithelial

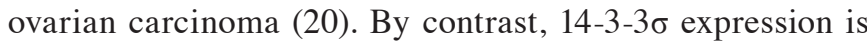
upregulated in head and neck squamous cell carcinoma (21) and pancreatic cancer (22). This data supports a dual role for $14-3-3 \sigma$ in the development of cancer.

In the present study, 14-3-3 $\sigma$ expression was significantly higher in gastric cancer compared with corresponding healthy gastric tissue. Immunohistochemical assays demonstrated that cases of gastric cancer exhibiting high 14-3-3 $\sigma$ expression levels also exhibited larger tumor volumes, indicating that $14-3-3 \sigma$ may be involved in gastric cancer proliferation. Furthermore, 14-3-3 $\sigma$ expression was correlated with TNM stage. This finding was consistent with those of a previous study conducted by Perathoner et al (23), which revealed a significant correlation between 14-3-3 $\sigma$ overexpression and tumor stage in colorectal carcinoma. These characteristics of 14-3-3 $\sigma$ expression may influence the prognosis of gastric cancer patients. With regard to the prognostic impact of 14-3-3 $\sigma$ in human cancer, positive 14-3-3 $\sigma$ overexpression was associated with a significantly decreased survival time compared with negative 
14-3-3 $\sigma$ expression in colorectal carcinoma (23). Consistent with this, the present study performed Kaplan-Meier survival analysis and multivariate Cox proportional-hazards regression analysis, which revealed that a high expression of 14-3-3 $\sigma$ was significantly associated with a poorer prognosis and shortened overall survival time.

In the present study, 14-3-3 $\sigma$ expression was correlated with tumor volume. Therefore, the association between 14-3-3 $\sigma$, and proliferation and apoptosis in gastric cancer was investigated. The results of the present study demonstrated a positive correlation between 14-3-3 $\sigma$ and $\mathrm{Ki}-67$, indicating that 14-3-3 $\sigma$ may be associated with the proliferation of gastric cancer cells. 14-3-3 $\sigma$ exerts anti-apoptotic effects by interacting with Bax, a pro-apoptotic protein (24). To investigate the role of 14-3-3 $\sigma$ in the apoptosis of gastric cancer cells, the correlation between 14-3-3 $\sigma$ and Bcl-2/Bax was explored. The positive rate of $\mathrm{Bcl}-2$ expression in the 14-3-3 $\sigma$-positive cases was significantly higher compared with the rate of Bcl-2 expression in the 14-3-36-negative cases. Furthermore, no correlation was identified between 14-3-3 $\sigma$ and Bax expression.

In conclusion, the present study investigated the expres-

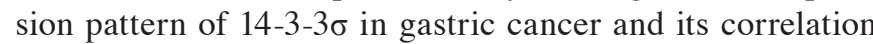
with specific clinicopathological features. The results of the present study indicate that 14-3-3 $\sigma$ may be involved in cell proliferation and apoptosis in gastric cancer. Furthermore, 14-3-3 $\sigma$ overexpression is associated with an unfavorable prognosis. Therefore, 14-3-3 $\sigma$ may be a promising target for the treatment of gastric cancer and, critically, investigations into the 14-3-3 $\sigma$-associated molecular mechanisms of gastric cancer cell proliferation and apoptosis should be conducted.

\section{Acknowledgements}

The present study was supported by grants from the National Natural Science Foundation of China (grant nos. 81302245 and 81201831).

\section{References}

1. Correa P: Human gastric carcinogenesis: a multistep and multifactorial process - First American Cancer Society Award Lecture on Cancer Epidemiology and Prevention. Cancer Res 52: 6735-6740, 1992.

2. Hara T, Ooi A, Kobayashi M, et al: Amplification of c-myc, K-sam, and c-met in gastric cancers: detection by fluorescence in situ hybridization. Lab Invest 78: 1143-1153, 1998.

3. Yu J, Miehlke S, Ebert MP, et al: Frequency of TPR-MET rearrangement in patients with gastric carcinoma and in first-degree relatives. Cancer 88: 1801-1805, 2000.

4. Nakatsuru S, Yanagisawa A, Ichii S, et al: Somatic mutation of the APC gene in gastric cancer: frequent mutations in very well differentiated adenocarcinoma and signet-ring cell carcinoma. Hum Mol Genet 1: 559-563, 1992.

5. Kim JH, Takahashi T, Chiba I, et al: Occurrence of p53 gene abnormalities in gastric carcinoma tumors and cell lines. J Natl Cancer Inst 83: 938-943, 1991.

6. Park K, Kim SJ, Bang YJ, et al: Genetic changes in the transforming growth factor beta (TGF- $\beta$ ) type II receptor gene in human gastric cancer cells: correlation with sensitivity to growth inhibition by TGF- $\beta$. Proc Natl Acad Sci USA 91: 8772-8776, 1994.
7. $\mathrm{Xu} \mathrm{HW}$, Ren F, Yu YM and Cai CZ: Runx3 expression in lymph nodes with metastasis is associated with the outcome of gastric cancer patients. Oncol Lett 2: 1275-1279, 2011.

8. Lalle M, Leptourgidou F, Camerini S, et al: Interkingdom complementation reveals structural conservation and functional divergence of 14-3-3 proteins. PLoS One 11: e78090, 2013.

9. Rosenquist M, Alsterfjord M, Larsson $\mathrm{C}$ and Sommarin $\mathrm{M}$ : Data mining the Arabidopsis genome reveals fifteen 14-3-3 genes. Expression is demonstrated for two out of five novel genes. Plant Physiol 127: 142-149, 2001.

10. Chan TA, Hermeking H, Lengauer C, et al: $14-3-3 \sigma$ is required to prevent mitotic catastrophe after DNA damage. Nature 401: 616-620, 1999

11. Laronga C, Yang HY, Neal C and Lee MH: Association of the cyclin-dependent kinases and 14-3-3 sigma negatively regulates cell cycle progression. J Biol Chem 275: 23106-23112, 2000.

12. Xiao T, Mi W, Li M, et al: Analyzed the molecular interaction network of tumor suppressor gene 14-3-3 sigma in lung cancer cell based on stable isotope labeling by amino acids in cell culture technology. Zhonghua Yu Fang Yi Xue Za Zhi 47: 752-756, 2013 (In Chinese).

13. Evren S, Dermen A, Lockwood G, et al: mTOR-RAPTOR and 14-3-3 $\sigma$ immunohistochemical expression in high grade prostatic intraepithelial neoplasia and prostatic adenocarcinomas: a tissue microarray study. J Clin Pathol 64: 683-688, 2011.

14. Zhang Z, Zhang G and Kong C: High expression of Cdc25B and low expression of $14-3-3 \sigma$ is associated with the development and poor prognosis in urothelial carcinoma of bladder. Tumour Biol 35: 2503-2512.

15. Liu S, Howell P, Ren S, et al: The 14-3-3o gene promoter is methylated in both human melanocytes and melanoma. BMC Cancer 27: 162, 2009.

16. Xie D, Sham JS, Zeng WF, et al: Heterogeneous expression and association of $\beta$-catenin, p16 and c-myc in multistage colorectal tumorigenesis and progression detected by tissue microarray. Int J Cancer 107: 896-902, 2003.

17. Ide M, Nakajima T, Asao T and Kuwano H: Inactivation of 14-3-3 $\sigma$ by hypermethylation is a rare event in colorectal cancers and its expression may correlate with cell cycle maintenance at the invasion front. Cancer Lett 207: 241-249, 2004.

18. Iwata N, Yamamoto H, Sasaki S, et al: Frequent hypermethylation of CpG islands and loss of expression of the 14-3-3 $\sigma$ gene in human hepatocellular carcinoma. Oncogene 19: 5298-5302, 2000.

19. Nakamura Y, Oshima K, Naoi Y, et al: $14-3-3 \sigma$ expression is associated with poor pathological complete response to neoadjuvant chemotherapy in human breast cancers. Breast Cancer Res Treat 134: 229-236, 2012.

20. Mhawech-Fauceglia P, Herrmann FR, Anderws C, et al: 14-3-3o expression and prognostic value in patients with epithelial ovarian carcinoma: a high throughput tissue microarray analysis. Eur J Surg Oncol 35: 763-767, 2009.

21. Erovic BM, Pelzmann M, Grasl MCh, et al: Mcl-1, vascular endothelial growth factor-R2, and 14-3-3 $\sigma$ expression might predict primary response against radiotherapy and chemotherapy in patients with locally advanced squamous cell carcinomas of the head and neck. Clin Cancer Res 11: 8632-8636, 2005.

22. Li Z, Dong Z, Myer D, et al: Role of $14-3-3 \sigma$ in poor prognosis and in radiation and drug resistance of human pancreatic cancers. BMC Cancer 10: 598, 2010

23. Perathoner A, Pirkebner D, Brandacher G, et al: 14-3-3o expression is an independent prognostic parameter for poor survival in colorectal carcinoma patients. Clin Cancer Res 11: 3274-3279, 2005

24. Guweidhi A, Kleeff J, Giese N, et al: Enhanced expression of 14-3-3sigma in pancreatic cancer and its role in cell cycle regulation and apoptosis. Carcinogenesis 25: 1575-1585, 2004. 